\title{
An Improved Leader Selection Approach for Improving Network QoS
}

\author{
Mahima \\ Research Scholar M.Tech \\ (CSE) \\ SITM, Sonepat
}

\author{
Vinay Kumar Nassa, Ph.D \\ Director-Principal \\ SITM, Sonepat
}

\author{
Maneela \\ Assistant Professor \\ Department of Computer \\ Science \& Engg. \\ SITM, Sonepat
}

\begin{abstract}
To improve the effectiveness and QoS of service in a sensor network there are number of communication and localization architectures followed by sensor network. One of such architecture is Leader Selection Architecture. This architecture restrict the communication to short distances so that the energy consumption of a node is reduces. In this paper, an improved approach is defined to perform the selection of Leader. This leader selection architecture approach is defined under multiple parameters including the energy, connectivity analysis and the balancing over the network. The improvement is also performed to generate the safe communication over the network controlled by the leader node. The obtained results show that the work has improved the network communication as well as network life.
\end{abstract}

\section{Keywords}

Leader Selection Architecture, LEACH, Hierarchical, Multihop

\section{INTRODUCTION}

A sensor network is a self organized wireless adhoc network defined with large number of sensing nodes along with energy constraint. These sensing nodes are defined with limited power and resources. There are number of real time applications where sensor network is implemented such as scientific research applications, statistical applications, disaster area application, war zones etc. According to the architectural organization and application, there are number of associated challenges with sensor network some of these challenges includes the node localization, communication hurdles, route optimization, security effectiveness, dynamic operations etc. The most standard form of sensor network architecture is composed with multiple sensor nodes and a base station. The server is also attached as the main component of outer environment to communicate the network information, outside the network. The standard form of network architecture is shown in Fig 1.

This structured network form is defined under some network architecture. One of such architecture is segmented architecture. In this architectural form, complete sensor network is divided in small area segments called Segments. Each Segment is having number of sensor nodes and a Leader. The sensor node performs communication with Leader and Leader performs the communication with base station. There are number of associated protocols that improve the effectiveness of segmented architecture. These protocols include LEACH, PEGASIS, and ESPDA etc. All these protocols support the concept of Leader Selection Architecture and aggregation.

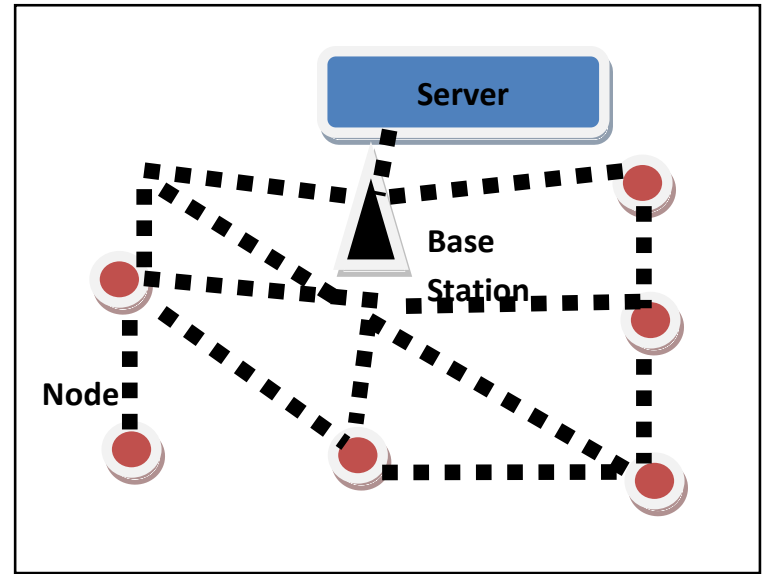

Fig 1: Sensor Network Architecture

These Segment based protocol are liable to perform reliable and efficient network communication. The Segmented network is further divided in two main networks

\subsection{Single-Hop Model}

In this network model, the Segment members perform direct communication with Leader. LEACH is such kind of protocol that supports single hop communication. This protocol is

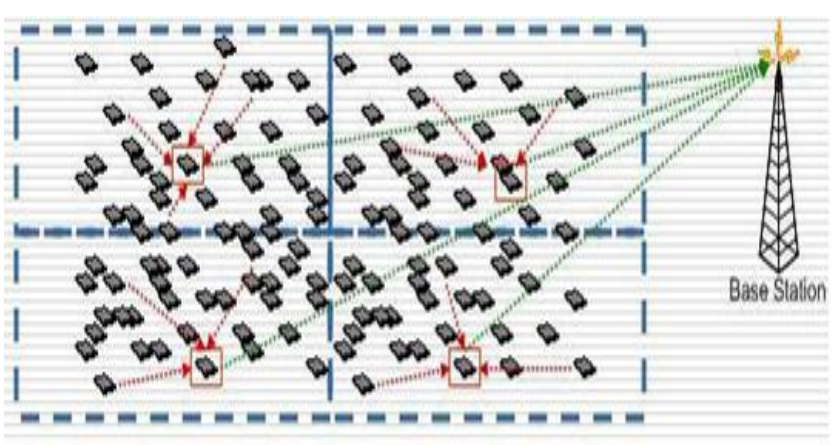

Fig 2: Single Hop Leader Selection Architecture

Further extended to LEACH C, LEACH E protocols. But these all protocol set support single level of Leader Selection Architecture. According to this protocol architecture, the nodes communication directly with Leader and Leader is capable to communicate directly with base station. The single hop Segment level communication is shown in Fig 2. 


\subsection{Multi-Hop Model}

As the name suggest, in this network architecture, the size of Leader is large. In such case, the Leader communication is based on the sensing range capability of network nodes. The closer nodes to Leader can perform direct communication with base station whereas the distance node can perform communication using intermediate node. The extension of LEACH protocol called LEACH M. This network architecture is shown in Fig 3.

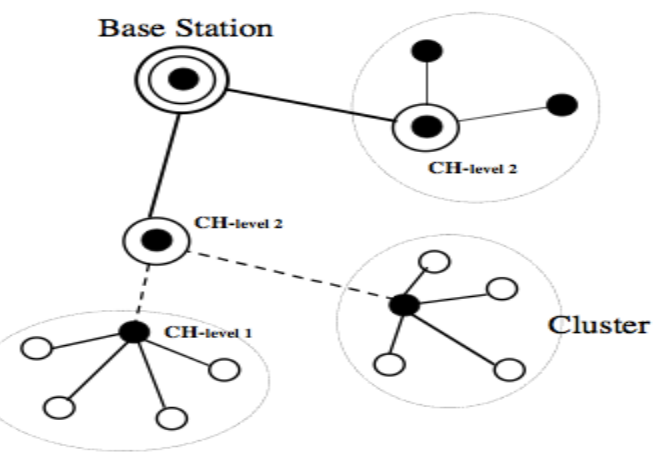

Fig 3 : Multi-Hop Segmented Model

In this paper, an exploration to the Leader Selection Architecture protocols is defined. The Segmented architecture and the standard Leader Selection Architecture model are discussed. In this section, a description to the network clustered architecture and its types are described and explored.

\section{LITERATURE REVIEW}

Lot of work is already defined by different researchers on Leader Selection Architecture and communication in sensor network. In this section, some of the work defined by earlier researchers is discussed.[1] Kamal Kumar Sharma, Dr. Harbhajan Singh and Dr. R.B Patel in their work, to detect congestion, each node calculates its node rank based on the parameters buffer Size, hop count, channel busy ratio and MAC overhead. When the node rank crosses a threshold value $T$, the sensor node will set a congestion bit in every packet it forwards. If the congestion bit is set, the downstream node calculates the Rate Adjustment Feedback based on the rank and propagates this value upstream towards the source nodes. The source nodes will adjust their transmission rates dynamically based on this feedback. [2] Basaran, KyoungDon Kang, Mehmet H. Suzer in their work "Hop-by-Hop congestion control technique and load balancing in Wireless Sensor Networks", used the Effective Queue Length (EQL) is measured. EQL is used to determine whether the congestion is occurred or not. The technique called CONSEQ (Control of Sensor Queues) is used to control the congestion. The CONSEQ aims to reduce congestion and, thereby, decrease the delay and energy consumption due to packet losses and retransmission in WSN. [3] Chieh-Yih Wan, Shane B. Eisenman,Andrew T. Campbell In "Energy efficient Congestion Detection and Avoidance in sensor networks, CODA, propose it as an energy efficient congestion control scheme for sensor networks was proposed. CODA (Congestion Detection and Avoidance) comprises three mechanisms: (i) receiver-based congestion detection; (ii) open-loop hop-by-hop backpressure; and (iii) closed-loop multi-source regulation. CODA detects congestion based on queue length as well as wireless channel load at intermediate nodes. Furthermore it uses explicit congestion notification approach and also an AIMD rate adjustment technique. [4]

Muhammad Monowar, ObaidurRahman, Al-Sakib Khan Pathan, and ChoongSeon Hong In "Prioritized Heterogeneous Traffic-oriented Congestion Control Protocol in Wireless Sensor Networks", proposes a Prioritized Heterogeneous Traffic-oriented Congestion Control Protocol (PHTCCP) to control congestion. It uses packet service ratio to detect congestion. Packet service ratio is defined as the ratio of average packet service rate and packet scheduling rate in each sensor node. PHTCCP uses hop-by-hop rate adjustment for controlling the congestion. The output rate of a node is controlled by adjusting the scheduling rate. [5] RekhaChakravarthi, C.Gomathy In "IPD: Intelligent Packet Dropping algorithm for congestion control in Wireless Sensor Networks", the congestion is detected by measuring the length of the queue. The queue length increases when the Packet inter-arrival time is more than Packet inter-service time. Using queue length, the Buffer Occupancy is calculated. When the Buffer Occupancy increases, the congestion increases. Congestion is controlled by assigning priority to the data packets. When the Buffer Occupancy increases, the data packets are dropped depending on priority assigned to the data packets i.e., Intelligent Packet Dropping. [6]

Hull B., Jamieson K., and Balakrishnan H. assumed that congestion is detected by measuring the queue length. The congestion is controlled by using three techniques i) hop-byhop flow control, ii) source rate limiting, and iii) prioritized MAC. Even in high offered load it claims to achieve good throughput and fairness. [7] Ee C. and Bajcsy R. in hop-byhop congestion control technique, Congestion Control and Fairness (CCF), which uses packet service time to infer the available service rate and therefore detects congestion in each intermediate sensor node. CCF ensures simple fairness.

However, it lacks efficient utilization of the available link capacity when some nodes do not have any traffic to send or nodes remaining in sleep mode or the nodes whose flows do not pass through the congested area. [8] R.Then Malar work PCCP is a recent congestion control protocol for WSNs which uses hop-by-hop approach for rate control. PCCP is a node priority based congestion control protocol which allows sensor nodes to receive priority-dependent throughput. However, PCCP does not have any mechanism for handling prioritized heterogeneous traffic originated from a single node. In [10], congestion is detected by calculating depth of congestion at the sink node. To calculate depth of congestion service and arrival rate are used. It's the ratio of local packet inter-service rate and local packet inter-arrival rate. Hop-byHop Rate control Technique (HRCT) is implemented to control congestion. The sensor/transmission rate of the node is adjusted based on depth of congestion and overall sensor priority. [9] B. Hull in his work Fusion is another congestion mitigation technique that uses queue lengths to detect congestion. Fusion uses three different techniques to alleviate congestion, viz, hop-by-hop flow control, rate limiting, and a prioritized MAC. [10]

ESRT was proposed by Yogesh SankarasubramaniamÖzgür B. Akan Ian F. Akyildiz which is based on an event-to-sink reliability model and provides reliable event detection without any intermediate caching requirements. ESRT also seeks to achieve the required event detection accuracy using minimum energy expenditure and has a congestion control component. [11] In this paper an efficient approach is presented for 
utilizing the aggregation of data in a Wireless Sensor Network and the assuring of end-to-end encryption of data between the leaves and sink. One of the goals of the paper was to minimize the bit transmission between the sensor nodes and therefore to find an efficient encryption algorithm which is simple to implement and in turn would prolong the life of batteries [11] in this paper, author proposes two protocols that consume less energy and maintain the privacy of the aggregated data. The results show that this protocol is better than the previous protocols if we talk in terms of energy utilization or the correctness of the aggregated data.

\section{PROPOSED WORK}

The presented work is about to identify a network leader so that the controlled and effective communication will be drawn over the network. The presented work is defined in a series of stages. In the earlier stage, complete network will be divided in smaller partitions and each partition will be controlled by a node called agent node. The agent node will perform the partition monitoring based on static and dynamic parameters. The static parameters include the distance, energy and connectivity parameters. The dynamic parameters include the communication and load analysis. Once the analysis will be performed, the agent node will identify the communicating nodes over the network. Based on these vectors, the leader node selection will be performed on the agent nodes. The node that is closer to the communicating nodes will be elected as the leader. The leader node will store the communicating path between the nodes and reduce the path election. The leader node will also be responsible for path generation so that effective communication will be drawn. The flow of presented work is shown in figure 4 .

\subsection{Functionality}

The working of leader selection process is defined in terms of stages. These stages include the Setup Stage and Steady Stage. During the Setup stage, the decision is taken regarding the formation of a Leader. It means it will check whether a node can be Leader or not. This decision is based on the residual energy of the node. The decision is also based on the basic of threshold specification. To elect a node as the Leader, each node broadcast an advertisement to show its intention. Once the requests are collected, the Leader selection is performed based on the signal strength and the position of node. During the Steady Stage, each Leader waits for receiving the data from all Segment nodes. As it gets the data, perform the aggregation and sent it to the base station. The functionality of Leader Selection Architecture is shown in Fig 4
Define a network with $\mathrm{N}$ sensor nodes

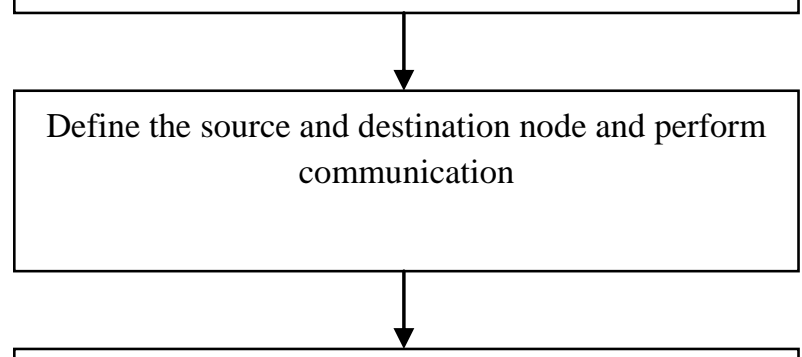

Divide the network in $\mathrm{N}$ segments and perform the communication analysis

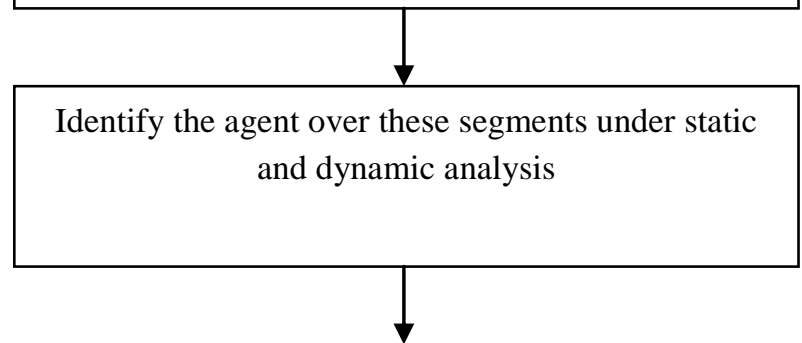

Identify the network area with high communication where control monitoring required

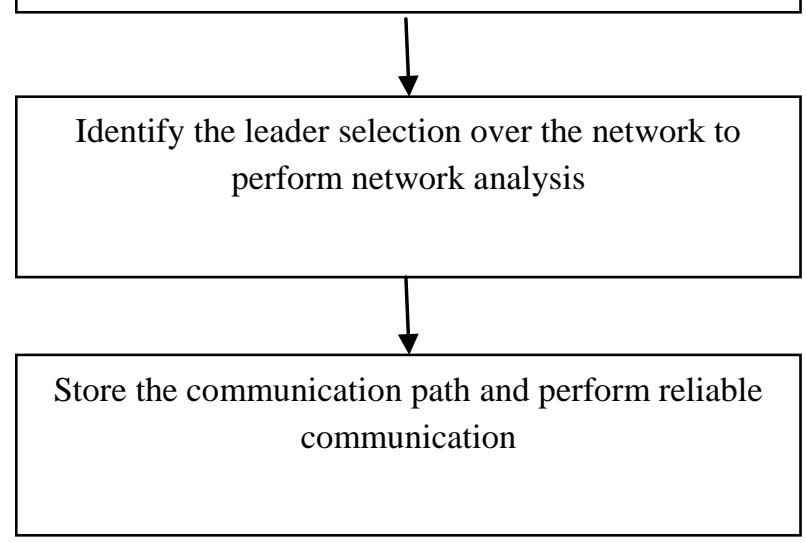

Fig 4: Flow of Work 


\section{RESULTS}

The presented work is implemented in mat lab environment with some defined network parameters. The parameters considered in this work are shown in the table 1 .

Table 1: Network Parameters

\begin{tabular}{|c|c|}
\hline Parameter & Values \\
\hline Number of Nodes & 75 \\
\hline Network Area & $800 \times 800$ \\
\hline Traffic & CBR \\
\hline Packet Size & $4 \mathrm{~KB}$ \\
\hline
\end{tabular}

The work is implemented in a network with fault or attack nodes over the network. The work is defined to identify the effective leader and performed the leader controlled reliable communication over the network. The analysis of the work is performed under network life and network communication parameters. These results are explained in this section. The first parameter considered here is network life that is described in terms of dead node and alive node analysis when the communication is performed over the network for 1000 rounds. The dead node comparative analysis of existing and proposed work is shown in table 2 .

Table 2: Dead Node Analysis (Existing vs. Proposed)

\begin{tabular}{|c|c|c|}
\hline Rounds & Existing & Proposed \\
\hline 0 & 0 & 0 \\
\hline 100 & 72 & 60 \\
\hline 200 & 73 & 63 \\
\hline 300 & 73 & 66 \\
\hline 400 & 73 & 68 \\
\hline 500 & 73 & 71 \\
\hline 600 & 73 & 71 \\
\hline 700 & 74 & 73 \\
\hline 800 & 74 & 73 \\
\hline 900 & 74 & 73 \\
\hline 1000 & & \\
\hline & & \\
\hline
\end{tabular}

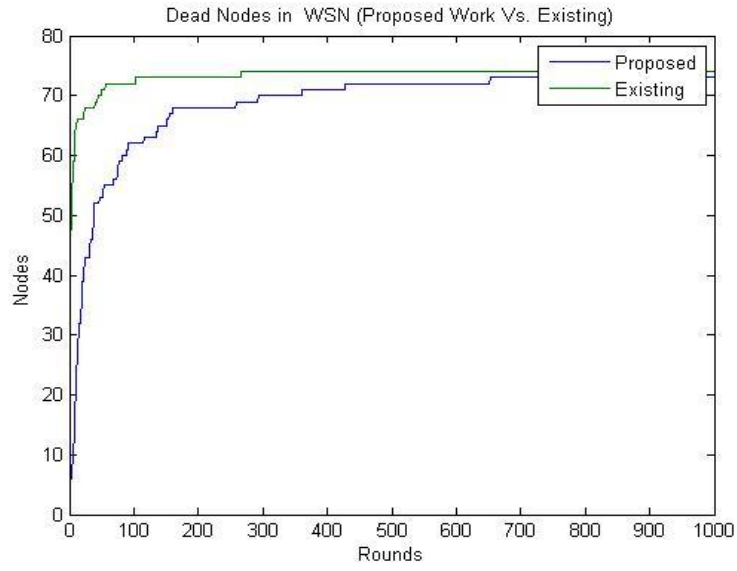

Fig 5 : Dead Node Analysis

Here Fig 5 is showing the comparative analysis of Dead Node process in case of existing and proposed approach. Here blue line is showing the results for proposed approach and green line is showing the results for existing approach. As we can see, in case of existing approach, node start losing energy earlier so that the energy loss ratio in existing approach is higher. Whereas, in proposed approach, the nodes will keep the energy for maximum time and network is alive for more number of rounds. In existing work 75 nodes are dead after completion of 1000 rounds whereas in case of proposed approach about 70 nodes are dead.

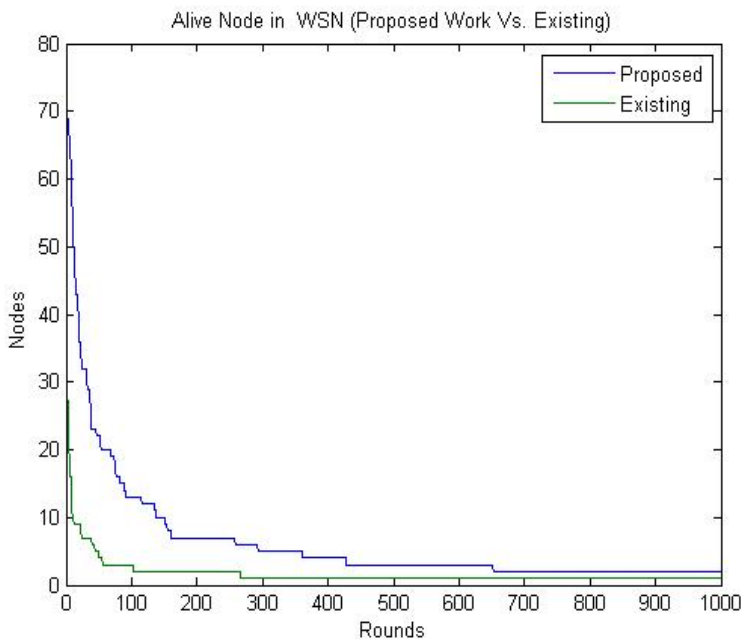

Fig 6 : Alive Node Analysis

Here Fig 6 is showing the comparative analysis of Alive Nodes in case of existing and proposed approach. Here blue line is showing the results for proposed approach and green line is showing the results for existing approach. As we can see, in case of existing approach, node start losing energy earlier so that the energy loss ratio in existing approach is higher. Whereas, in proposed approach, the nodes will keep the energy for maximum time and network is alive for more number of rounds. In case of existing approach about 1 node are alive after completion of 1000 rounds whereas in case of proposed approach about 5 nodes are alive. 
Table 3: Communication Analysis (Existing vs. Proposed)

\begin{tabular}{|c|c|c|}
\hline Rounds & Existing & Proposed \\
\hline 0 & 0 & 0 \\
\hline 100 & 809 & 2626 \\
\hline 200 & 1124 & 3869 \\
\hline 300 & 1424 & 4934 \\
\hline 400 & 1724 & 5853 \\
\hline 500 & 2024 & 6686 \\
\hline 600 & 2324 & 7413 \\
\hline 700 & 2624 & 7951 \\
\hline 800 & 2888 & 8451 \\
\hline 900 & 3088 & 8880 \\
\hline 1000 & 3288 & 9280 \\
\hline & &
\end{tabular}

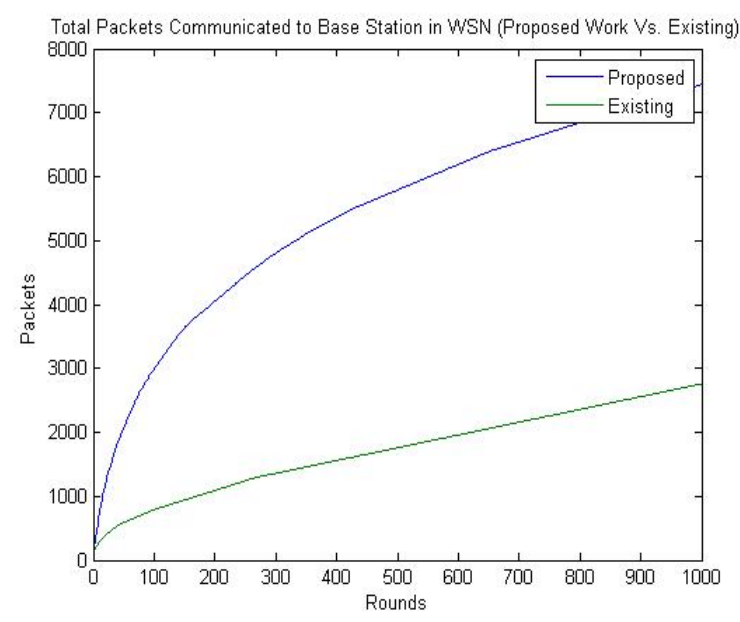

Fig 7 : Network Communication Analysis

Here Fig 7 is showing the comparative analysis of total communicating packets over the network. The figure is showing the aggregative communication analysis for all nodes over the network. Here blue line is showing the results for proposed approach and green line is showing the results for existing approach. As we can see, in case of existing approach, the packet communication is much lesser than proposed approach because, the nodes are losing energy very fast and it reduced the network life.

\section{CONCLUSION}

In this paper, an improved algorithmic approach is defined to perform the leader selection for sensor network as well as to perform the leader controlled communication over the network. The paper has presented the approach along with functionality as well as presents the analysis results obtained from the work under different parameters. The analysis is here performed under network life and communication parameters. The comparison of presented work is performed with basic leader selection approach without handling the network fault. The obtained shows that the work has provided the significant improvement in terms of network life and communication.
The work is here performed on the statistical analysis and it can be improved in future under some optimization approach.

\section{REFERENCES}

[1] C. Chong, S. Kumar and B. Hamilton, "Sensor networks: Evolution, opportunities and challenges," Proceedings of the IEEE, 9(18), pp.247-256, 2003

[2] Kamal Kumar Sharma, Dr. Harbhajan Singh and Dr. R.B Patel "A Hop by Hop Congestion Control Protocol to Mitigate Traffic Contention in Wireless Sensor Networks", in Proceedings of International Journal of Computer Theory and Engineering, December, 2010.

[3] Basaran, kyoung-Don Kang, Mehmet H. Suzer "Hop-byHop Congestion Control and Load Balancing in Wireless Sensor Networks", in proceedings of 2010 IEEE $35^{\text {th }}$ conference on Local Computer Networks, 2010.

[4] Chieh-Yih Wan, Shane B. Eisenman, Andrew T. Campbell," Energy-Efficient Congestion Detection and Avoidance in Sensor Networks ", in proceedings of ACM Transactions on Sensor Networks2011.

[5] Muhammad Monowar, ObaidurRahman, Al-Sakib Khan Pathan, and ChoongSeon Hong "Prioritized Heterogeneous Traffic-Oriented Congestion Control Protocol for WSNs" in proceedings of The International Arab Journal of Information Technology, 2012.

[6] RekhaChakravarthi, C.Gomathy "IPD: Intelligent Packet Dropping Algorithm for Congestion Control in Wireless Sensor Network", in Proceedings of IEEE, 2010.

[7] Hull B., Jamieson K., and Balakrishnan H., "Mitigating Congestion in Wireless Sensor Networks," in Proceedings of the 2nd International Conference on Embedded Networked Association for Computing Machinery Sensor Systems, USA, , 2004.

[8] Ee C. and Bajcsy R., "Congestion Control and Fairness for Many-to-One Routing in Sensor Networks," in Proceedings of the 2nd International Conference on Embedded Networked Association for Computing Machinery Sensor Systems, USA.

[9] R.Then Malar "Congestion Control in Wireless Sensor Networks Based Multi-Path Routing in Priority Rate Adjustment Technique “, in proceedings of International Journal of Advanced Engineering \& Applications, 2010.

[10] Rekha Chakravarthi, C. Gomathy "Hop-by-Hop Rate Control Technique for Congestion Due to Concurrent Transmission in Wireless Sensor Network" in proceedings of World of Computer Science and Information Technology Journal (WCSIT), 2011.

[11] C. Y. Wan, A. T. Campbell and L. Krishnamurthy, IPSFQ: A Reliable Transport Protocol for Wireless Sensor Networks," In Proc. WSNA 2002, September 2002, Atlanta, GA, USA.

[12] B. Ruzena and E.C. Tien, "Congestion Control and Fairness for Many-to-One Routing in Sensor Networks," ACM SENSYS, pp.148-161, 2004.

[13] C. Wang, B. Li and K. Sohraby, "Upstream Congestion Control in Wireless Sensor Networks through Crosslayer Optimization," IEEE Journal on Selected Areas in Communications, vol.25, no.4, pp.786-795, 2007. 